\title{
Miniaturized Reactors in Combinatorial Catalysis and High-Throughput Experimentation
}

\author{
Torsten Zech*, Peter Claus ${ }^{a}$, and Dieter Hönicke
}

\begin{abstract}
Different approaches to study the advantages and challenges of miniaturized reactor systems for combinatorial catalysis and high-throughput experimentation are presented. These are a parallel microchannel reactor, a chip-based 'single microchannel' reactor, a monolithic reactor acting as multichannel reactor, and a chip reactor design for liquid-phase processes. These developments are discussed in detail regarding their design, manufacturing and corresponding catalyst preparation issues. A number of applications are explained in conjunction with the description of a system for spatially resolved high-throughput analytics.
\end{abstract}

Keywords: Chip reactor · Combinatorial catalysis · High-throughput experimentation · Microchannel reactor · Miniaturization

\section{Introduction}

The miniaturization of chemical and physical processes and their integration onto microchips for specific applications are well known. Already today, the emerging high-throughput screening of combinatorial libraries has revolutionized drug discovery in the same way that microchips influenced the epochal development of computers and electronics. The pharmaceutical industry is, indeed, the main driver of the development of process miniaturization and integration.

Obviously, there are strong analogies between the development of combinatorial chemistry for drug discovery and combinatorial material science, including combinatorial catalysis as an important part. It is well worthwhile to read the excellent paper

\footnotetext{
${ }^{*}$ Correspondence: T. Zech

hte Aktiengesellschaft

Kurpfalzring 104, D-69123 Heidelberg, Germany

Tel: +4962217497172

Fax: +4962217497134

E-Mail: torsten.zech@hte-company.de

aTU Darmstadt, Institut für Technische und Makromolekulare Chemie

Petersenstrasse 20, D-64287 Darmstadt, Germany

bTU Chemnitz, Lehrstuhl für Technische Chemie

D-09107 Chemnitz, Germany
}

of Lebl [1] about the development of combinatorial chemistry and to compare it with today's situation in combinatorial material science and high-throughput experimentation. The first papers in combinatorial chemistry were published in the mid eighties. Since those days exponentially growing activities in this field have been observed, fundamentally changing the old research paradigms. The first companies operating in this field were founded in the late eighties and the beginning of the nineties. Combinatorial chemistry and high-throughput screening are nowadays core technologies in all major pharmaceutical companies. In 1995 a landmark publication of Xiang et al. [2] was published extending the application of combinatorial chemistry to the discovery of solid state materials.

Since then a large number of applications of combinatorial methods to catalysis has been published. These papers propose that high-throughput experimentation had the potential to substitute totally or at least partially the time - and man power - consuming conventional screening methods. This can also be regarded as the motivation for the ongoing research in two screening stages, viz. catalyst discovery as the first stage and catalyst optimization as the second one. In both stages, the application of either shaped catalyst bodies used in a multitude of parallel fixed beds or catalytically active walls of several microflow channels in parallel is useful. Screening results obtained with the former are appropriate, in particular, for scalable catalyst preparation procedures for industrial applicable fixed bed catalysts. Their preparation is carried out predominantly by well-known conventional methods, e.g. impregnation, precipitation, ion-exchange, grafting. Screening results from the latter are directly applicable to microchannel reactors and catalytic wall reactors in general. The preparation techniques used include a wide variety of surface treatment and deposition methods, e.g. sputtering, CVD, PVD, chemical and electrochemical deposition, plasma-chemical and anodic oxidation, sol-gel deposition.

During the last three years a number of library screening techniques for the different stages in the development of heterogeneous catalysts have been published [3-10]. However, little is known about continuously operated miniaturized devices for the application in high-throughput discovery programs in catalysis or in process development in general. In contrast to conventional bench-scale systems, highthroughput screening and the application of 
very small amounts of materials are the two major advantages of screening approaches using miniaturized chemical devices. Recently, a very interesting contribution was published by Jensen and co-workers [11], who used a so-called miniaturized crossflow chemical reactor with a very short packed-bed that is also suitable for kinetic studies. The authors state that this type of reactor could also be applied for highthroughput testing of heterogeneous catalysts. However, an example of this is not given. Another approach was explored by Bergh et al. [12] who used an arrangement of reaction chambers with thin or thick film catalysts on a flat substrate for parallel catalyst testing. A method for continuously supplying reactants to the reaction chambers is also described and claimed [13]. However, the description of the actual design of the reactor is rather fuzzy, making the evaluation of this approach from an engineering point of view difficult. The term 'microreactor' is also used by Senkan et al. [10], although conventional catalyst particles with a diameter of several millimeters are used and no microstructured parts are included in the reactor design.

Therefore, the aim of this contribution is to review our approaches for continuously operated microchemical systems for high-throughput experimentation and to discuss advantages and challenges for the application of such systems in catalyst screening and the estimation of process parameters.

\section{Parallel Development of Microchannel Reactors}

\subsection{Experimental Setup}

\subsubsection{Reactor Module and Microstructured Inlays}

For the parallel screening and development of catalysts for heterogeneously catalyzed reactions in microchannel reactors a system design imitating the conditions usually found in microchannel reactors is required. Therefore, a reactor module was developed [14] consisting of a stack of metallic frames, as shown in Fig. 1. Stacking these metallic frames together, several parallel and independent microreaction chambers are formed. The prototype shown in Fig. 1a consists of 35 microreaction chambers. Each chamber can hold a microstructured inlay carrying one catalyst as a catalytically active coating on the microchannels.

In order to test the catalytic properties of the coated catalysts, the reactants, supplied by a number of mass flow controllers (Brooks), are uniformly distributed to all parallel microreaction chambers. The reactants flow through the microchannels of the microstructured inlays (see Fig. 1b), react on the catalytically active surface of the microchannels and the products are transported through the product outlets at the end of each microreaction chamber having a cross section of $2 \times 0.5 \mathrm{~mm}$.

The position of the inlay in the metallic frame is shown in detail in Fig. 2. It is obvious that the fabrication of the frames and the inlays requires the use of high precision machining in order to reduce bypasses. Furthermore, precautions have to be taken to allow easy removal and effective remounting of the inlays.

The module was heated externally to achieve reaction temperatures up to $450{ }^{\circ} \mathrm{C}$ in the study described here. The design of the reactor module still has room for improvement, e.g. by designing the module as a crossflow heat exchanger [15] to ensure a constant temperature along the reaction channels or by using other materials such as ceramics, allowing operation at high temperatures. The reactor module design al- lows the use of replaceable microstructured catalyst inlays made of different materials such as metals, silicon, ceramics, and glass. Based on this flexibility, many different catalyst synthesis procedures as well as microchannel geometries can be applied. Therefore, the idea of using such a reactor module and replacable microstructures has recently been anticipated by other researchers [16][17].

\subsubsection{Spatially-resolved Product Analysis}

The product outlets of the reactor module are spaced only by a thin metallic layer of a thickness of $200 \mu \mathrm{m}$ (compare Fig. 1a). This compact module design requires a powerful, spatially resolved method to analyze the product compositions of one catalyst with low interference from adjacent microreaction chambers.

For that reason, a sampling device was developed [14][18], shown schematically in Fig. 3. The device consists of a capillary probe that can be micropositioned in xyzdirection within the parallel reactor config-

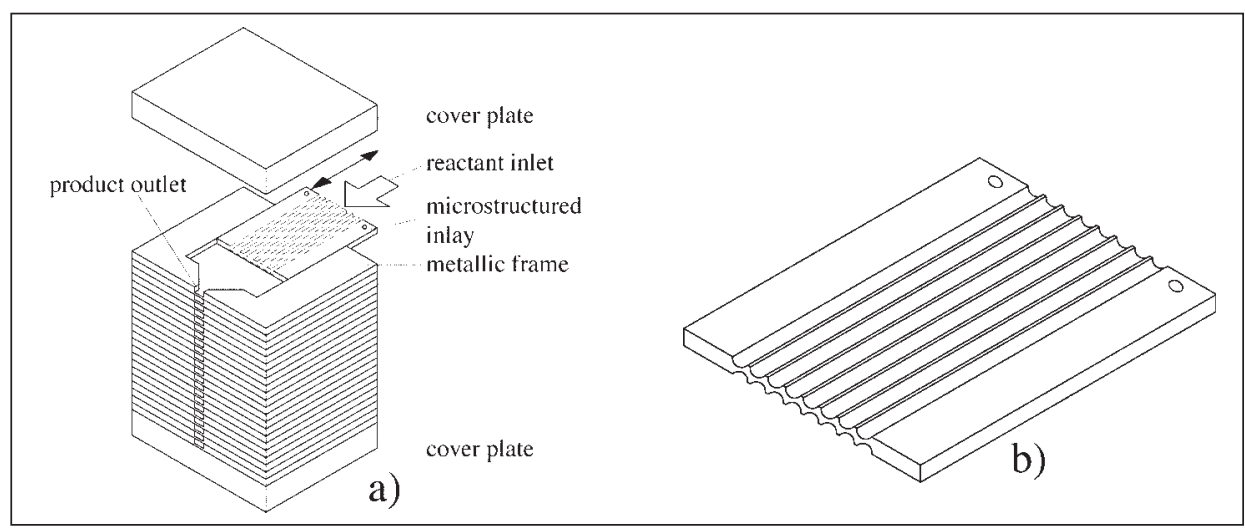

Fig. 1. a) Schematic view of the reactor module consisting of a stack of metallic frames. The catalyst inlays are mounted and removed in the directions of the arrow. b) A microstructured catalyst inlay has an overall size of $20 \mathrm{~mm} \times 16 \mathrm{~mm}$ and is made of aluminum by wet etching (80 microchannels, channel radius: $130 \mu \mathrm{m}$ ).
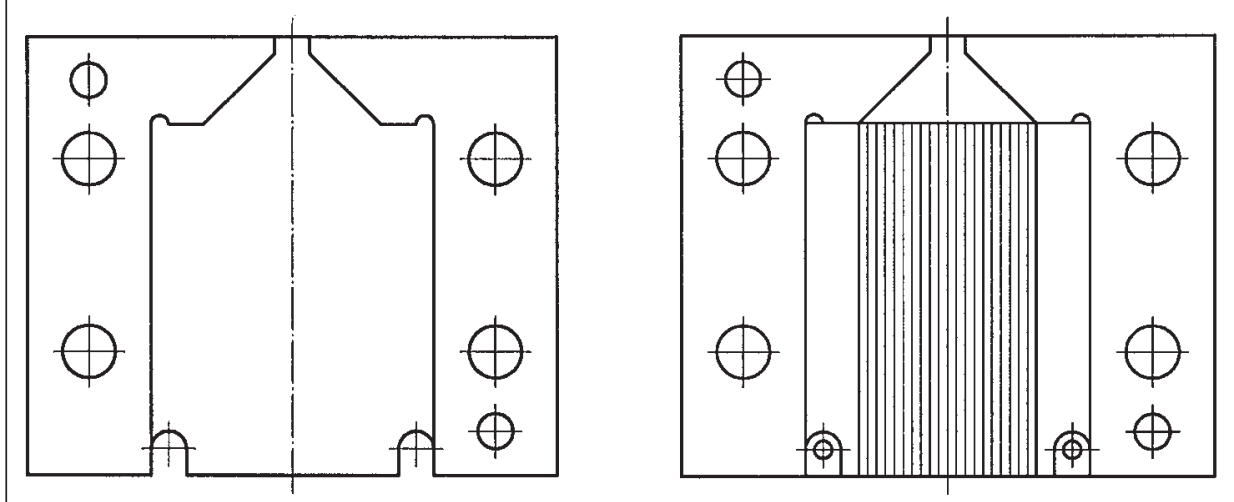

Fig. 2. Schematic view of a single metallic frame with and without a mounted microstructured inlay. 
uration. Furthermore, the capillary probe is connected to and transfers the samples to the analytical instrument of choice, e.g. a mass spectrometer, a gas chromatograph, or a gas chromatograph with MS-coupling. In the present study, a conventional, commercially available mass spectrometer (Hiden Analytical, Warrington, UK) was used. The gas samples were continuously transferred to the mass spectrometer through the capillary allowing a fast online analysis.

The capillary can be positioned with high accuracy under hazardous chemical conditions and high thermal demands. During the sampling the capillary moves first in the $\mathrm{x}$ - and $\mathrm{y}$-direction to the corresponding outlet of a microreaction chamber and af- terwards in the $\mathrm{z}$-direction to a defined depth into the product outlet of that chamber. The parameters and the position of the capillary are adjusted and supervised by a CCD camera equipped with magnifying optics and are freely programmable. The materials of the housing and the sealing were carefully chosen to withstand temperatures up to $450{ }^{\circ} \mathrm{C}$. The analysis speed can be determined by choosing the right MS conditions and the application of short and small transfer capillaries. Depending of course on the nature of the products to be investigated, analysis times of less than 60 s can easily be achieved.

Based on our experience with this analysis tool, the sampling device in com-

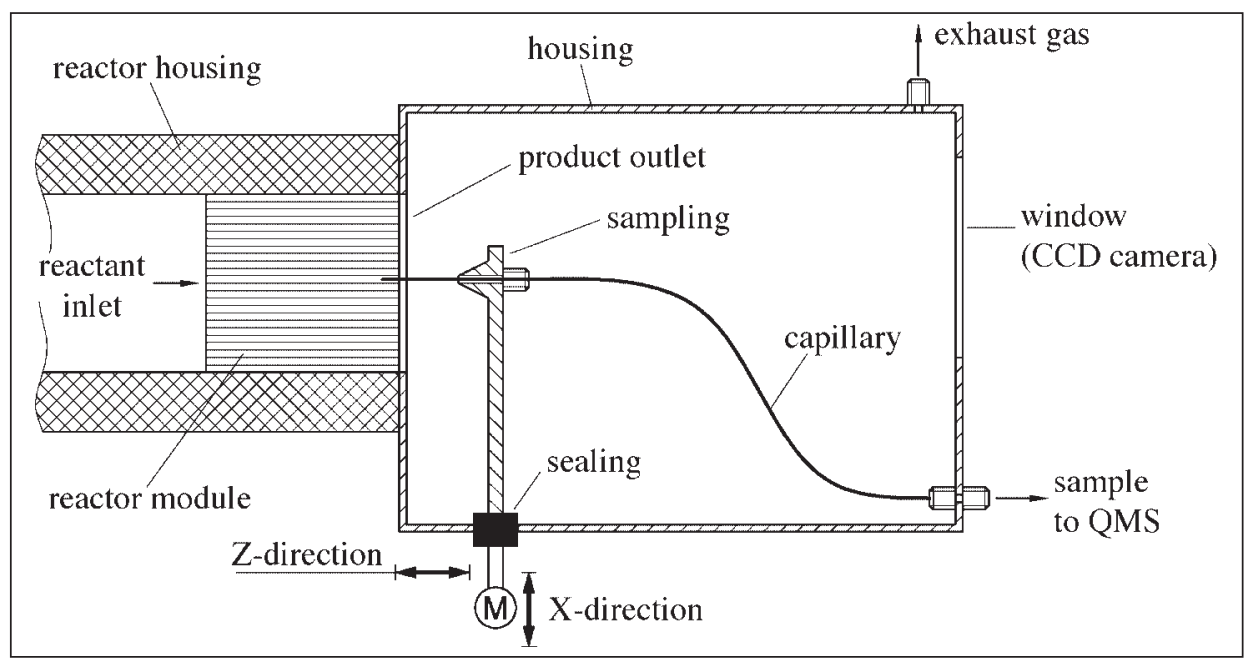

Fig. 3. Sketch of the sampling device used for the parallel microchannel reactor. The gas samples are taken sequentially by the xyz-positioned capillary probe and transferred to the analytical instrument, e.g. to a mass spectrometer.

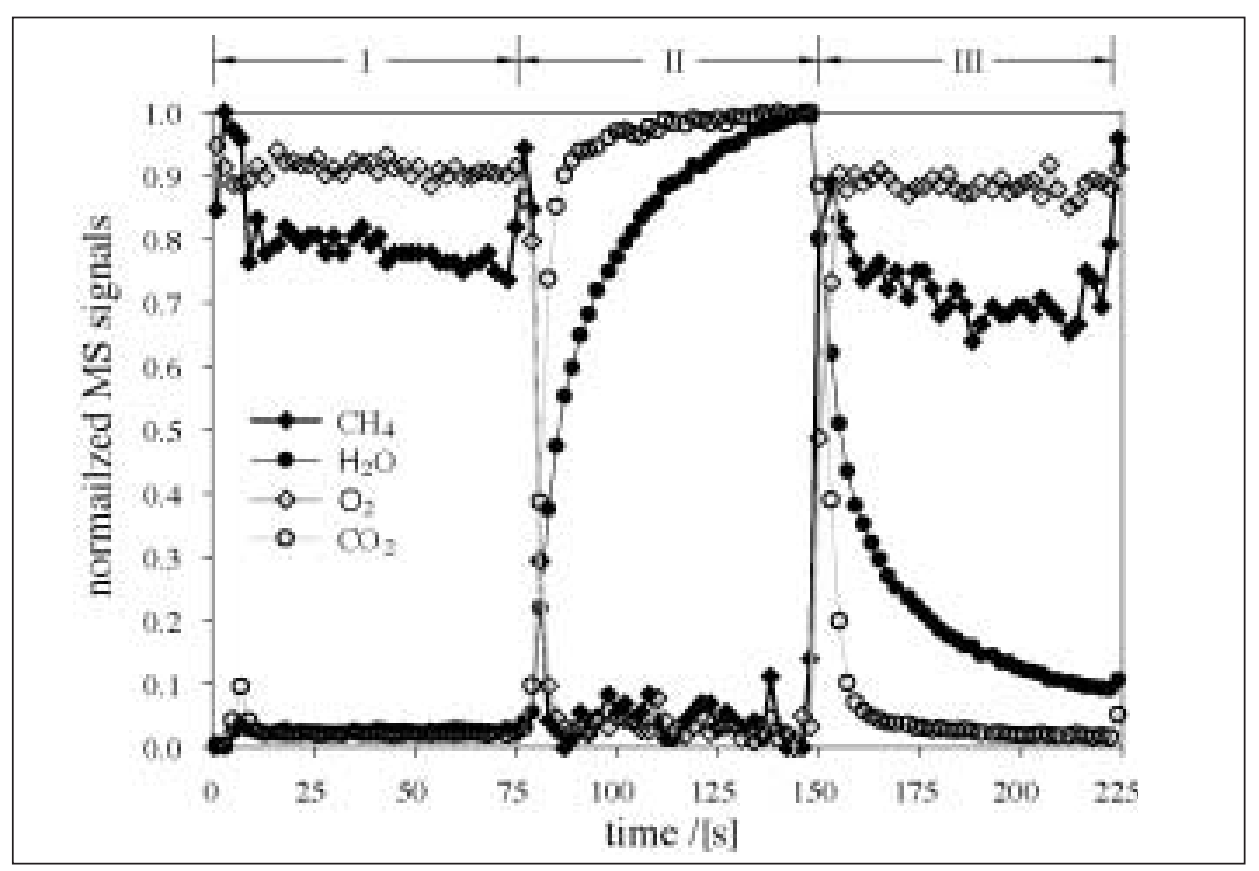

Fig. 4. Trend of the normalized MS-signals during the sequential screening of three adjacent catalysts in a typical experiment: methane oxidation, $450{ }^{\circ} \mathrm{C}, 5.6$ vol- $\% \mathrm{CH}_{4}, 2.8$ vol- $\% \mathrm{O}_{2}, \mathrm{Ar}$ balance, 1.1 bar. bination with an appropriate analysis technique can be regarded as an almost universal analysis tool for gas-phase reactions in combinatorial catalysis. It can be used with conventional analytical equipment and virtually every reactor configuration and application requiring time and spatially resolved sampling and the analysis of complex gaseous mixtures. The presented anaylsis technique was quickly adapted by other groups [10].

\subsubsection{Screening Procedure}

Results from the validation of the presented catalyst screening unit are shown in Fig. 4. The figure illustrates the trend of the normalized MS-signals during the sequential screening of three adjacent catalysts (indicated by I, II, and III) for methane oxidation activity at $450{ }^{\circ} \mathrm{C}$.

It can be observed clearly that the concentrations of the corresponding products change very quickly when moving the capillary from one reaction chamber to the next $(\mathrm{t}=0-10 \mathrm{~s}$ and $\mathrm{t}=75-80 \mathrm{~s})$. The catalyst in reaction chamber II shows a rather high conversion degree, indicated by the decrease of the signals for $\mathrm{CH}_{4}$ and $\mathrm{O}_{2}$ as well as by the increase of the signals for $\mathrm{CO}_{2}$ and $\mathrm{H}_{2} \mathrm{O}$. A new steady state is reached within a few seconds. In contrast to these observations, the MS signal for $\mathrm{H}_{2} \mathrm{O}$ does not reach a steady state within the given analysis time. This behaviour is probably due to adsorption effects of $\mathrm{H}_{2} \mathrm{O}$ within the transfer capillary and the mass spectrometer itself. This implies that the rate limiting step in a quantitative analysis of the product mixture (as well as for the whole screening procedure) is the analysis of the $\mathrm{H}_{2} \mathrm{O}$ in the product stream. A screening protocol optimized for a maximum sample rate (e.g. in primary screening) would probably exclude $\mathrm{H}_{2} \mathrm{O}$ from a detailed analysis, as $\mathrm{H}_{2} \mathrm{O}$ is usually not the product of interest.

However, the example illustrates that a routine analysis within about $60 \mathrm{~s}$ is possible resulting in 60 catalytic tests per hour. Furthermore, it can be concluded that a number of factors have to be considered when carrying out fast analytics for the different stages of a catalyst screening process and the limits of the analytical method have to be taken into account.

\subsection{Partial Hydrogenation of 1,3-Butadiene}

\subsubsection{Catalyst Preparation}

To illustrate the application of the screening system in detail, the partial hydrogenation of 1,3-butadiene was chosen as a model reaction [19]. Two catalyst libraries (Lib 1 and Lib 2) were prepared. For Lib 1, a porous alumina coating on top 


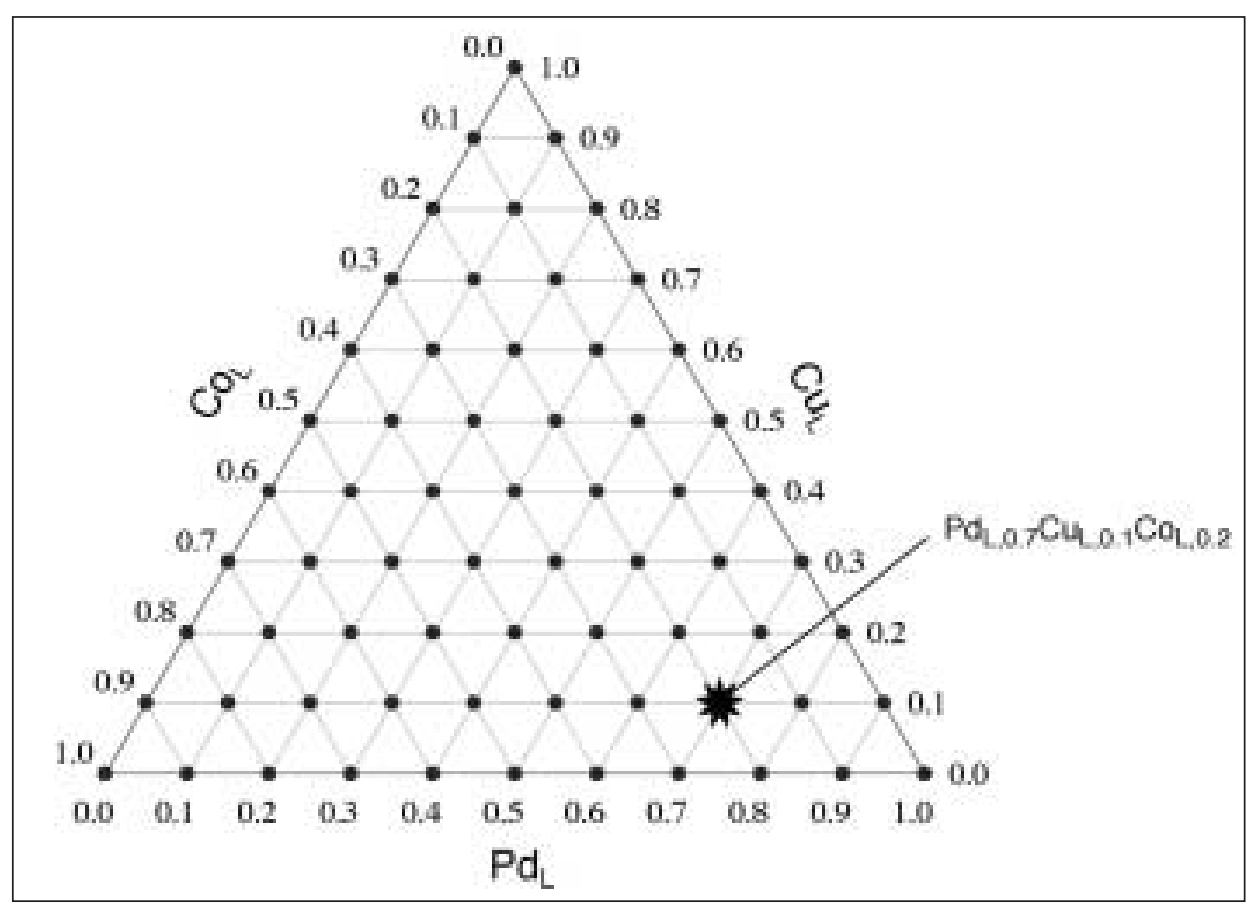

of the microchannels of the microstructured inlays with a thickness of $15 \mu \mathrm{m}$ was prepared by anodic oxidation in oxalic acid. This process yields to an $\mathrm{Al}_{2} \mathrm{O}_{3}$ layer as catalyst support with very uniform pores of a diameter of about $30 \mathrm{~nm}$. The mass of $\mathrm{Al}_{2} \mathrm{O}_{3}$ per inlay is about $22 \mathrm{mg}$. For the preparation of the second library, Lib 2, sulfuric acid was used as the electrolyte in the anodic oxidation. The resulting pore diameter is approximately $15 \mathrm{~nm}$ while the thickness and the mass of the $\mathrm{Al}_{2} \mathrm{O}_{3}$ layer were the same.

A wet impregnation procedure was chosen to deposit the catalytically active components into the pores of the $\mathrm{Al}_{2} \mathrm{O}_{3}$ layer. $\mathrm{Pd}, \mathrm{Co}$, and $\mathrm{Cu}$ were chosen as the catalytically active components [20][21]. The concentration of these components in the impregnation solutions were varied according to the ternary diagram shown in Fig. 5. The corresponding acetyl acetonates were used as precursors. In all experiments, the total concentration of the metal precursors was kept constant at $37.5 \mathrm{mg} / \mathrm{ml}$. The impregnation was carried out during $24 \mathrm{~h}$ at $25^{\circ} \mathrm{C}$. Chloroform was used as solvent. Finally, the catalysts were calcined for $18 \mathrm{~h}$ at $450{ }^{\circ} \mathrm{C}$ in air.

This preparation procedure resulted in 132 different catalysts ( 2 supports, 66 compositions). The catalyst preparation procedure was carried out manually but in parallel for 35 catalysts. This is acceptable as it was not the rate limiting step in the screening process.

\subsubsection{Screening Protocol}

The protocol for the screening of the prepared catalysts in the partial hydrogena- tion of 1,3-butadiene is shown in Table 1. First, the reactor was heated to $150^{\circ} \mathrm{C}$ to reduce the catalysts for $2 \mathrm{~h}$ in an $\mathrm{H}_{2}$ flow. After cooling the reactor to $50{ }^{\circ} \mathrm{C}$, the catalysts were screened under seven different reaction conditions by varying the residence time and the reactor temperature. Under each reaction condition, the catalysts were screened for $4 \mathrm{~h}$. With a cycle time of $\mathrm{t}_{\text {cyc }}=75$ min this means that each of the 35 catalysts was screened three times under the same reaction conditions in order to observe potential activation and deactivation phenomena. Having finished the screening protocol, a period of about $3 \mathrm{~h}$ is needed to cool down the reactor and mount a new set of catalysts. Thus, the whole screening protocol requires about $36 \mathrm{~h}$. In this basic study nine of the 35 reaction chambers contained inactive reference samples. In a single 1,3-butadiene, cycle time $t_{\mathrm{cyc}}=75 \mathrm{~min}$.

Fig. 5. Composition of samples containing Pd, $\mathrm{Cu}$, and $\mathrm{Co}$ prepared by wet impregnation according to a ternary diagram. $\mathrm{Pd}_{\mathrm{L}}, \mathrm{Co}_{\mathrm{L}}$, and $\mathrm{Cu}_{\mathrm{L}}$ represent the concentration of the metal precursor in the impregnation solution.

screening run, 546 catalytic tests are performed (735 including the reference samples).

\subsubsection{Catalytic Results}

A typical result of the screening of the catalysts from a single screening run is shown in Fig. 6 as a plot of the respective conversion degree. All reference samples show very similiar behaviour. Therefore, the number of reference samples could be reduced to increase the number of catalytic tests per time. The results for selected reaction conditions for the two catalyst libraries are shown in Fig. 7. In the ternary diagrams, the value of the yield to the partial hydrogenation products, the n-butenes, is plotted at the corresponding position of the catalyst. Furthermore, the plots contain isolines connecting points with the same mul-

Table 1. Screening protocol for the investigation of catalysts for the partial hydrogenation of

\begin{tabular}{lcrc} 
& $\begin{array}{c}\dot{\mathrm{V}}_{\text {total,STP }} \\
{[\mathrm{ml} / \mathrm{min}]}\end{array}$ & $\begin{array}{c}\mathrm{T} \\
{\left[{ }^{\circ} \mathrm{C}\right]}\end{array}$ & $\begin{array}{c}\text { Duration } t \\
{[\mathrm{~h}]}\end{array}$ \\
\hline $\begin{array}{l}\text { Heating } \\
\text { Reduction in } \mathrm{H}_{2}\end{array}$ & 150 & 1 & 2 \\
Cooling & 120 & 150 & 2 \\
\hline Screening with variation of parameters & 500 & 50 & 4 \\
& 400 & 50 & 4 \\
& 300 & 50 & 4 \\
& 200 & 50 & 4 \\
& 100 & 50 & 4 \\
Cooling and mounting of new catalysts & 500 & 50 & 4 \\
\hline Total & 500 & 100 & 3 \\
\hline
\end{tabular}




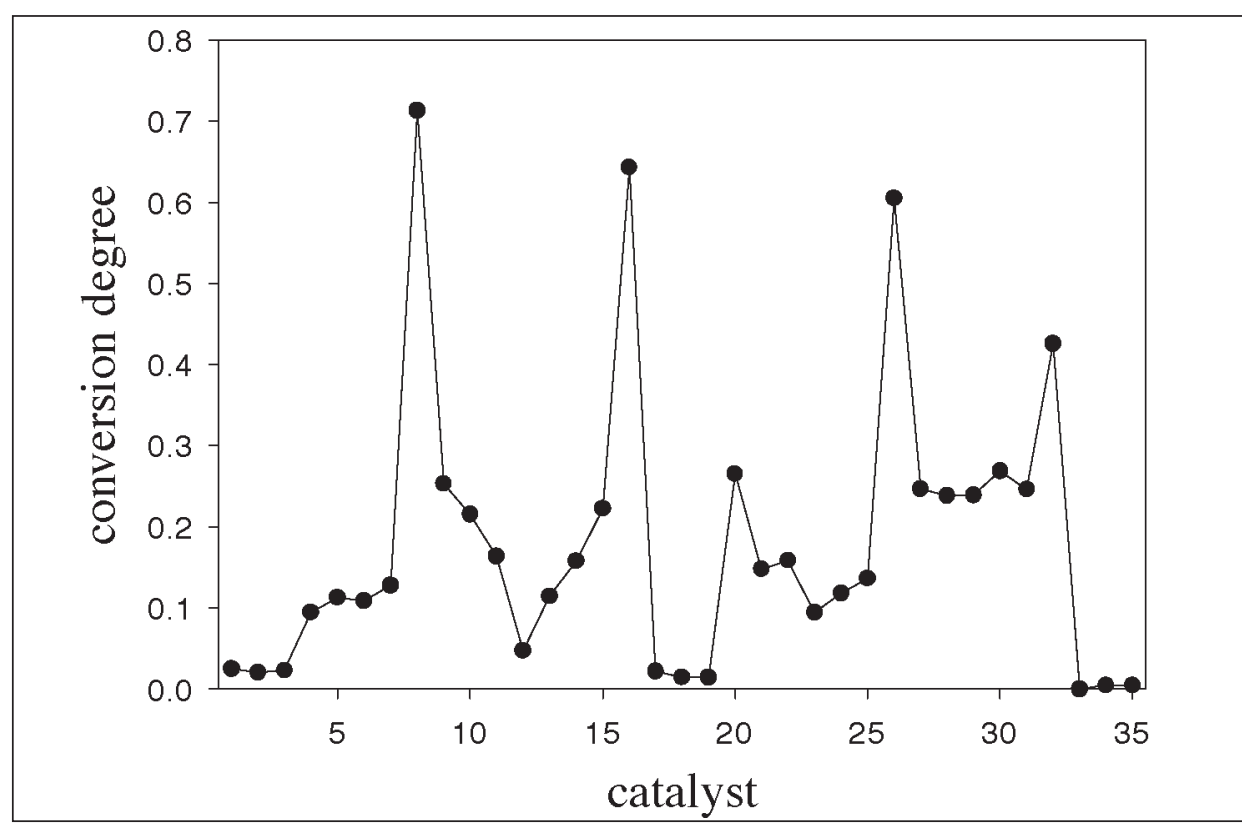

Fig. 6. Typical screening results of catalysts for the partial hydrogenation of 1,3-butadiene. The reaction chambers at the positions $1-3,17-19$, and $33-35$ contain inactive reference samples.

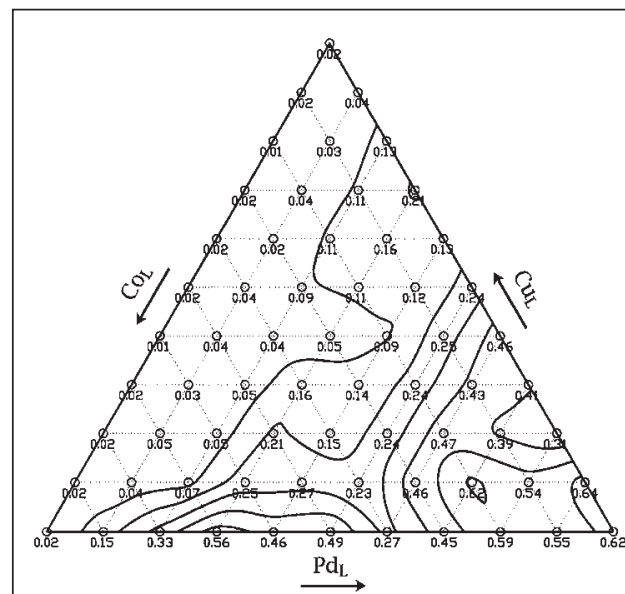

(a)

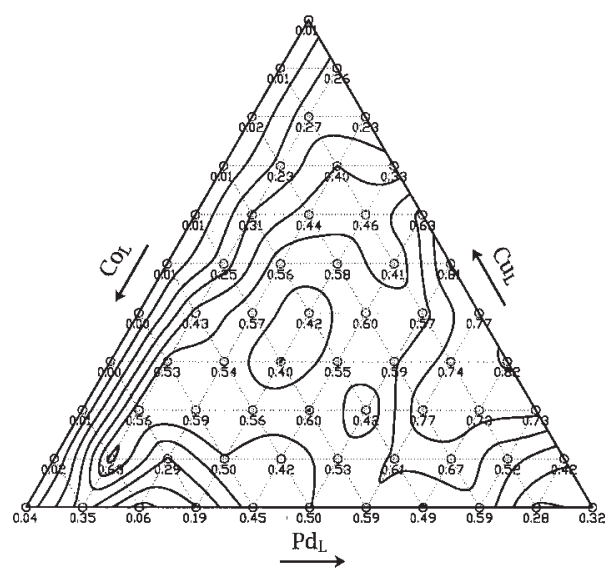

(c) (b)

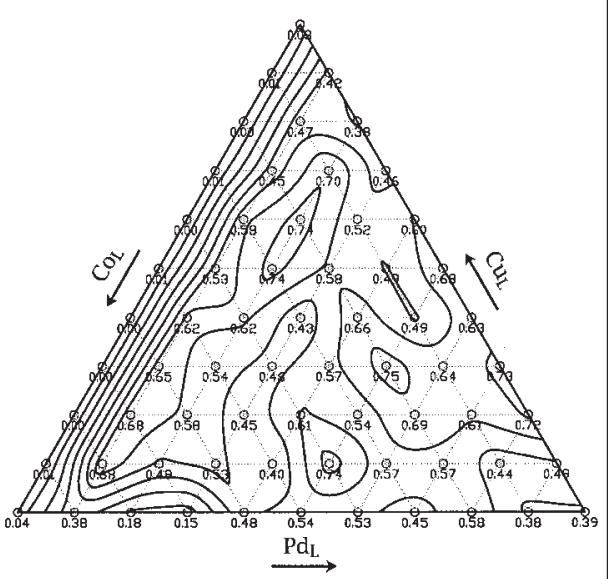

(d)

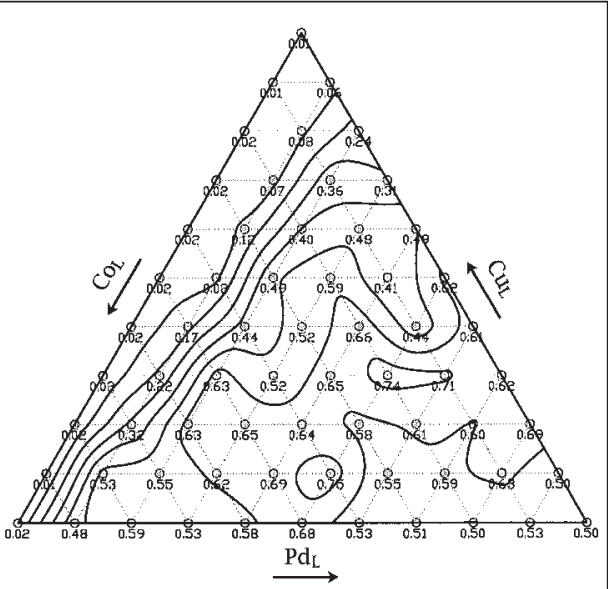

Fig. 7. Exemplary results from the screening of 132 different $\mathrm{Pd}-\mathrm{Co}-\mathrm{Cu}$ catalysts in the partial hydrogenation of 1,3-butadiene; Reaction conditions: $\mathrm{c}_{\mathrm{C} 4 \mathrm{H} 6}=1.6$ vol- $\%, \mathrm{C}_{\mathrm{H} 2}=1.6 \mathrm{vol}-\%, \mathrm{Ar}$ balance; precursor concentrations: see Fig. 5; a) Lib $1, T=50^{\circ} \mathrm{C}, \tau=60 \mathrm{~ms}$; b) Lib 2, $T=50^{\circ} \mathrm{C}$, $\tau=60 \mathrm{~ms} ; \mathrm{c}) \operatorname{Lib} 2, T=50^{\circ} \mathrm{C}, \tau=300 \mathrm{~ms}$; d) Lib $2, T=130^{\circ} \mathrm{C}, \tau=60 \mathrm{~ms}$. tiple of 0.1 . It has to be mentioned that it was not possible to distinguish the different butenes with the mass spectrometer used. Therefore, the cumulative selectivity to all n-butenes as well as the conversion degree of 1,3-butadiene was used to calculate the yield.

The yields of n-butenes for the catalysts of Lib 1 and Lib 2 at a reactor temperature of $\mathrm{T}=50{ }^{\circ} \mathrm{C}$ and a residence time of $\tau=$ $60 \mathrm{~ms}$ for Lib 1 and Lib 2 are compared in Fig. 7a and Fig. 7b. Obviously, the binary combinations of $\mathrm{Co}$ and $\mathrm{Cu}$ are inactive in this reaction for both libraries. An increase of the amount of palladium increases the yield to n-butenes. For Lib 1, the highest yields are above 0.6 and can be observed only at very high $\mathrm{Pd}$ concentrations. For Lib 2, the maximum yield reaches 0.75 at much lower Pd concentrations. In this experiment, very high Pd concentrations result in very active catalysts producing butane, thus reducing the yield of n-butenes. Moreover, it can be observed that ternary combinations generally outperform binary combinations of active components as well as the single components.

Referring to Fig. 7c, an increase of the residence time to $\tau=300 \mathrm{~ms}$ has a considerable effect on the yield of 1,3-butadiene. The yield over Pd-rich catalysts is reduced due to higher conversion degrees and lower selectivities. However, binary Pd$\mathrm{Cu}$-catalysts with a maximum of $82 \%$ yield perform better than binary $\mathrm{Pd}-\mathrm{Co}$-catalysts. Fig. 7d illustrates the impact of the temperature on the catalysts of Lib 2 . At a reaction temperature of $\mathrm{T}=130{ }^{\circ} \mathrm{C}$ the $\mathrm{Pd}$-content can be considerably reduced to achieve the same yields with $\mathrm{T}=50{ }^{\circ} \mathrm{C}$. Furthermore, the increase of the temperature reduces the yield for catalysts with high Pd-loadings. Again, this is due to lower selectivities to nbutenes at higher temperatures.

\section{3. 'Single Microchannel' Screening Devices}

\subsection{Motivation and Strategy}

As known from high-throughput screening applications in combinatorial chemistry, assay miniaturization can be one of the major economic drivers and present a significant competitive advantage. This is possibly also true for other areas of chemical research, e.g. combinatorial materials science and high-throughput experimentation.

Chip-based systems in particular seem to have a number of advantages that are worth exploring in high-throughput experimentation systems. These advantages are, for instance, efficient thermal control and 
short response times. By reducing the size of the libraries it becomes necessary to use high precision engineering technologies for both the catalyst preparation and the reactor fabrication. This may present a significant economic advantage in the long term. Miniaturization is also followed by a standardization of the screening workflow facilitating the comparison between library members and the reproducibility of the experiments. The challenges, however, are the catalyst design, the sampling and the product analysis as well as the integration of microchemical and electronic components on a chip. In addition, it has to be ensured that catalytic results gained on chip-basis remain scalable and can be compared with experiments on a larger scale.

A possible strategy for deploying the chip-based approach is illustrated in Fig. 8 [22]. Starting with a microfabrication step, a number of parallel microchannels are fabricated onto a flat substrate, e.g. a silicon wafer. Then, micropipetting systems applied, for instance, for the fabrication of DNA chips can be employed for catalyst synthesis via solution-based methodologies. The main property compared to the approach in section 2 is that a different catalytic material is prepared in each microchannel, resulting in so-called 'single microchannel' screening devices. Followed by a calcination and a bonding step the device is then ready for parallel catalytic tests and the corresponding high-throughput analytics.

\subsection{Reactor Layout and Fabrication}

Applying the described strategy, a number of 'single microchannel' screening devices was fabricated onto silicon according to the wafer layout shown in Fig. 9 [23]. In total, 120 reactors, grouped to chips with 8 or 16 reactors per chip varying the channel geometry, could be fabricated this way. The parallel microchannels have cross sections of, for instance, $500 \mu \mathrm{m} \times 200 \mu \mathrm{m}$. To ensure equal reactant distribution a socalled 'binary tree' manifold consisting of very small channels was used. The manifold was designed in a way that the resulting manifold channel length from the entry point of the reactants to the single microchannel reactors as well as the number of crossing points, channel cross sections etc. is equal for all reactors. Thus, the pressure drop of the flowing fluids acts as a regulator for the fluid distribution, ensuring equal distribution due to the geometric uniformity. Having passed the manifold, the fluids flow through the microchannels, react on the catalytic active surface and leave the chip separately through small holes on the back of the chip, where they can be an-

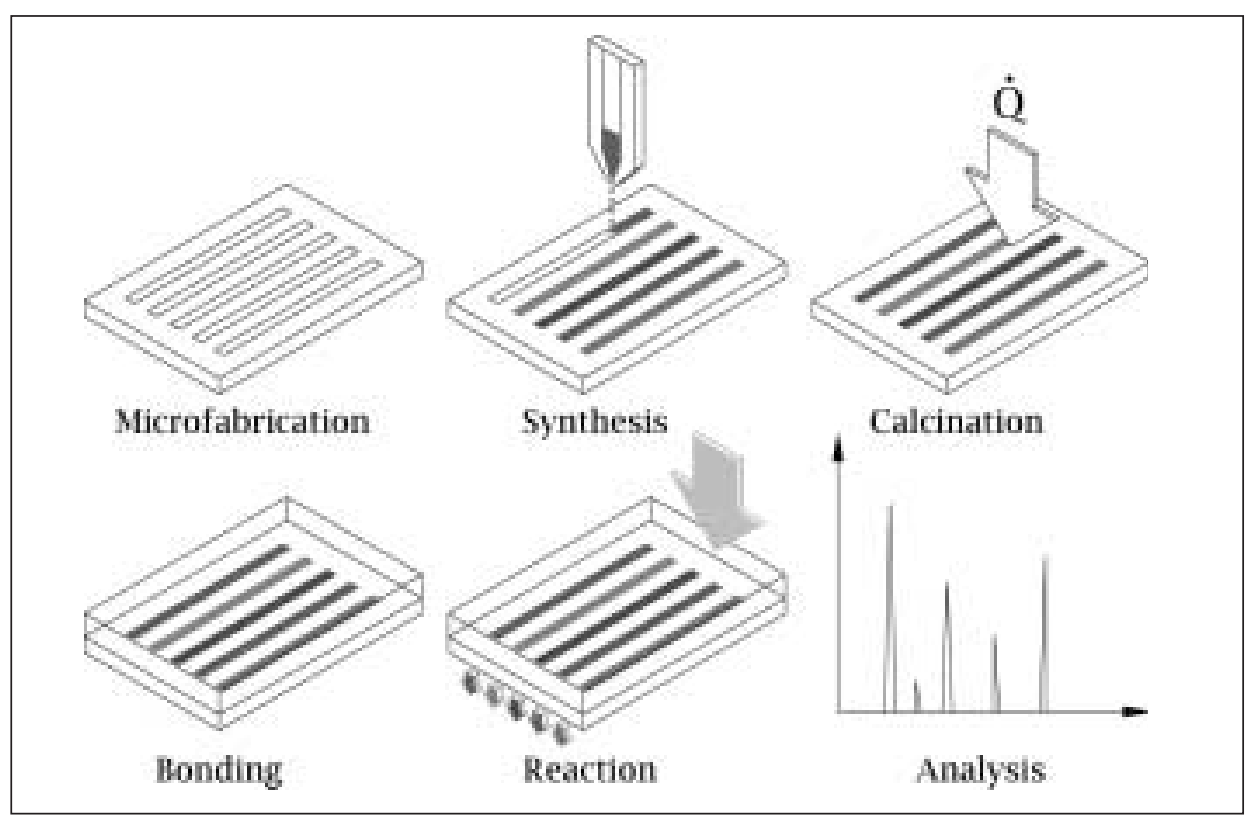

Fig. 8. Strategy for deploying substrate based 'single microchannel' screening devices

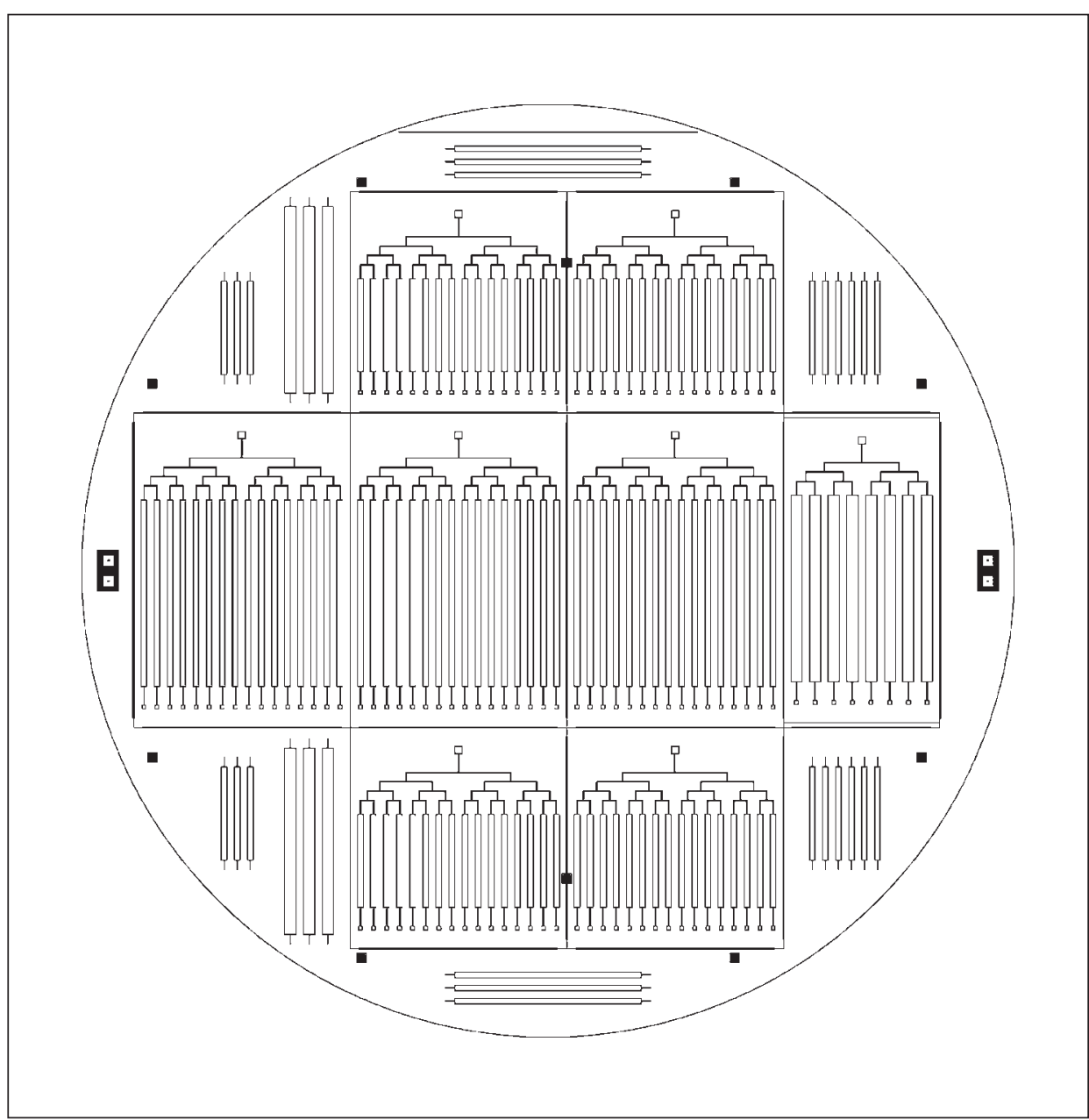

Fig. 9: Layout for 'single microchannel' screening devices on a $100 \mathrm{~mm}$ silicon wafer. The process yields eight chip-based parallel reactors (in total 120 reaction chambers) with different channel lengths and channel widths. 
alyzed by mass spectrometry as described in detail above.

\subsection{Catalyst Preparation in Single Microchannels}

In our experience it is important to stress that the greatest challenge is to develop a general and reproducible catalyst preparation method for single microchannels. Many experiments were carried out to automatically dispense solutions generated by the sol-gel technique to synthesize catalytic supports as coatings inside the single microchannels using a piezo-driven nanopipetting robot (GeSiM, Dresden, Germany, Fig. 10). Due to a number of problems with pipette clogging, droplet size, droplet direction and surface tension, this process could not be carried out fully automated. Instead, the coating of the microchannels via the sol-gel technique was done manually using the smallest available

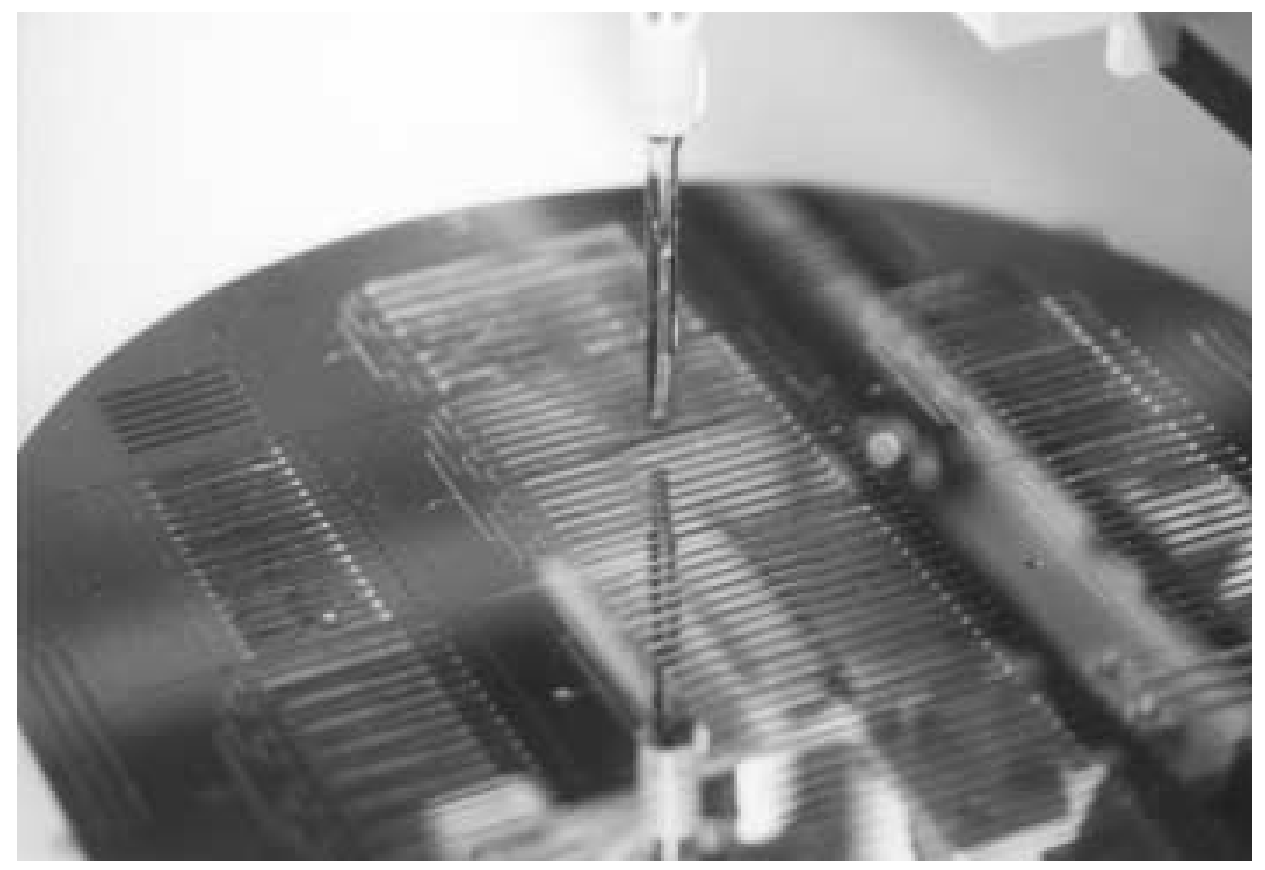

Fig. 10. Catalyst preparation by spatially resolved deposition of different precursor solutions through nanopipetting robots
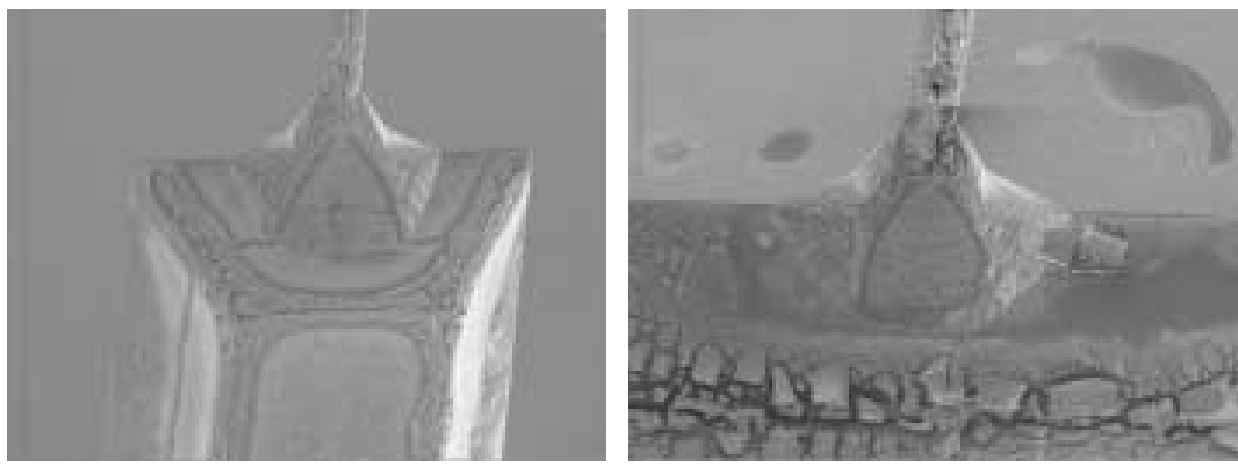

Fig. 11. REM-analysis of sol-gel- $\mathrm{SiO}_{2}$-coatings of single microchannels prepared by manually depositing precursor solutions into the microchannels of the study, namely automatic catalyst preparation, faster catalytic tests with less expenses in labour and material, and smooth screening workflow, could not be achieved. We report this approach here because it raised a number of questions that gave birth to new ideas and led to the development of the so-called 'single bead reactors' that solved almost all problems observed with the 'single microchannel' screening devices [24-26].

\section{Screening of Fixed-bed Catalysts in Monoliths as Multichannel Reactors}

For the fast parallel screening of heterogeneous catalysts also reactor systems based on the application of a ceramic monolith can be used where each channel represents a single fixed-bed reactor. Note that in this case, and contrary to the use of monoliths as washcoats for exhaust gas catalysts, a monolith has to be applied which is impermeable for gases. The gas composition produced by the catalytic reaction in a particular channel of the monolith can be analyzed sequentially by fast gaschromatographic analyses or a quadrupole mass spectrometer (QMS). For that purpose, the QMS inlet capillary is moved in $\mathrm{x}$ - and y-direction of the catalyst array and then into the particular channel (z-direction) by a three-dimensional (3D) positioning system based on the experiences of Zech et al. [14]. The position of the capillary above a monolith channel can be automatically changed by using the three stepping motors of the positioning system supervized by a CCD camera and exactly controlled by the software. Compared to a former application of monoliths as reactor module for fast catalyst screening [23] we achieved two important improvements of such a system: (i) Whereas in a former experience different catalytic materials were filled in the channels in the form of powder or catalyst particles only [23] we are now able to coat each channel with a particular catalyst comprising of one or more metals and/or oxides on a suitable support (e.g. $\mathrm{SiO}_{2}, \mathrm{TiO}_{2}, \mathrm{ZrO}_{2}$ ) [27]. (ii) In previous studies the test of the first channel was completed at least two hours after the gas composition of last channel was analyzed. Thus, it could be possible that deactivation phenomena falsify the catalytic activity and selectivity before the second analyses of the first channels could be examined. This could be eliminated by a special design of the gas inlet/outlet sytem coupled with the channel entrance. The new system was successfully applied to the total and partial oxidation of hydrocarbons [27]. 


\section{Chip Reactor for Liquid-phase Process Development}

\subsection{Field of Application}

Another interesting field of application for continuously operated miniaturized parallel reactors are liquid-phase reactions. The application prospects are broad for both the screening of homogeneous catalysts in the liquid phase and the development of liquid and multiphase processes on the microscale. While the screening of homogeneous catalysts mainly uses miniaturization as a tool and would basically apply the same methodology as presented above for heterogeneous catalysts (identical reactors in parallel, equal distribution of reactants etc.), the approach for developing liquid or potentially multiphase processes (with or without a catalyst) may be different. The miniaturized reactor size can indeed be the size on which the production of, for instance, specialty or hazardous chemicals is envisioned.

\subsection{Reactor Layout and Fabrication}

A flexible tool for changing the reactor configuration and reaction conditions is needed. In conjunction with this demand it is obvious that the reactor geometry is one of the basic factors for the functionality of the system. However, as far as can be judged from the literature, this question has not yet been addressed efficiently. To deal with this problem, a novel chip-based microreaction system has been developed [28]. The basic structure of such a chip reactor is shown in Fig. 12. The chip reactor itself consists of an arrangement of 19 parallel, independent microreaction systems on a single chip substrate, e.g. a silicon wafer. In the microreaction systems depicted in the figure, a two-stage chemical reaction can be performed: In reaction zone 1, the first two reactants are mixed and react with each other inside a microchannel. In reaction zone 2, a third reactant is introduced to react with the reaction product of zone 1 , again inside a microchannel. Finally, the reactants reach zone 3 , where the reaction is quenched as the result of a decrease of the temperature. The microreaction systems are all differently designed varying channel widths $(75-300 \mu \mathrm{m})$, total channel lengths $(10-100 \mathrm{~mm})$, mixing angles as well as the ratios of the corresponding reactor volumes in zone 1 and 2. Furthermore, all three reactants can be mixed in a single step (system 17), or there may be more than one position, where the reaction product of zone 1 is fed into the stream of the third reactant in zone 2 (systems 15, 16, 19).

The microreaction systems were fabricated by reactive ion etching of a $100 \mathrm{~mm}$

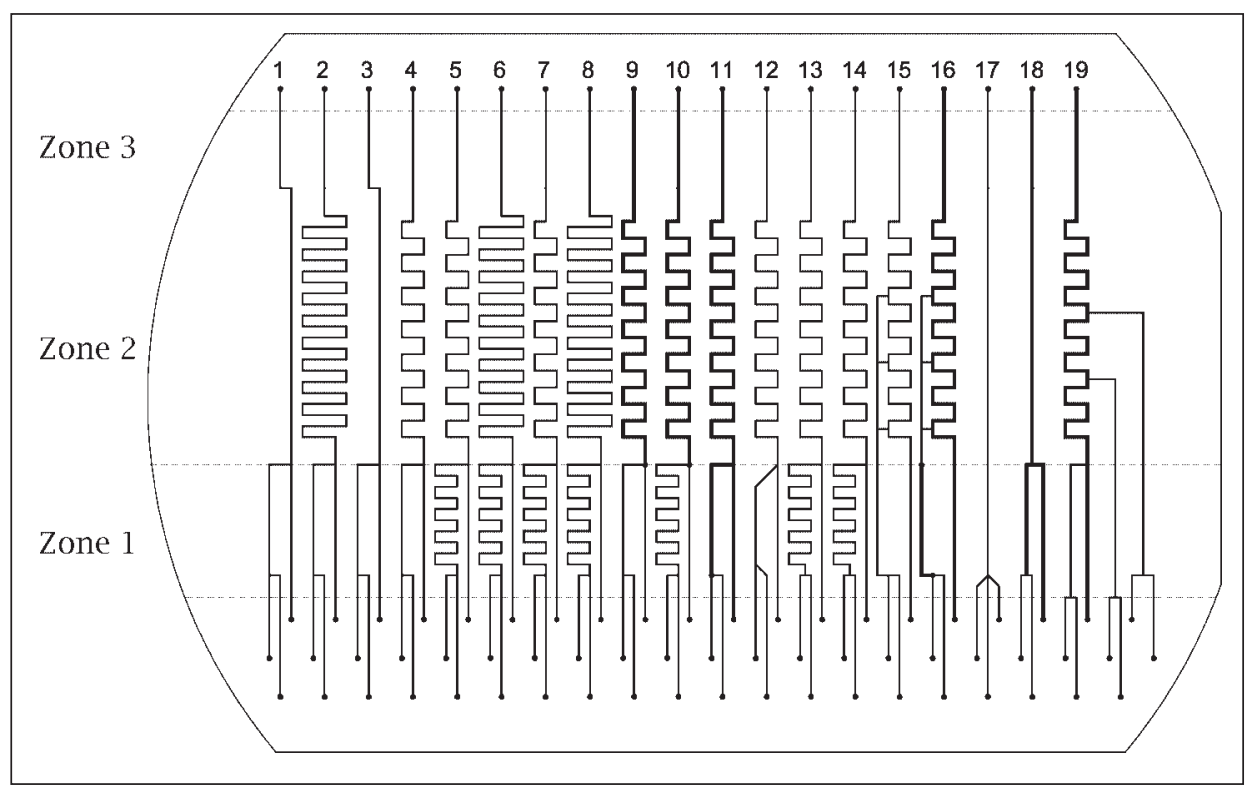

Fig. 12. Chip reactor: Parallel arrangement of 19 different two-stage microreaction systems with varying channel lengths and widths on a single $100 \mathrm{~mm}$ wafer

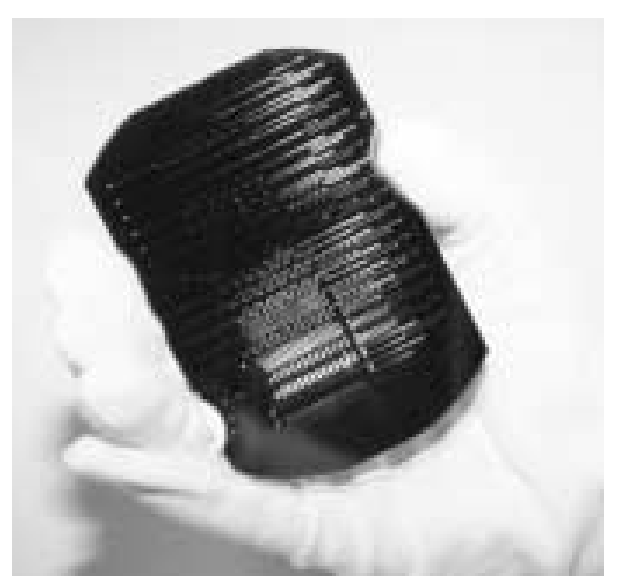

silicon wafer. All channels are $200 \mu \mathrm{m}$ deep. To gain fluidic access to the channel systems, holes into the start and end point of the channels were etched by applying a $\mathrm{KOH}$-etching process from the backside of the wafer. Then, the wafer was thermally oxidized to generate a chemically inert $\mathrm{SiO}_{2}$ coating inside the channels. Finally, the wafer was bonded to a Pyrex wafer to close the channel systems while keeping the chance to observe the processes inside the channels optically. A photograph of the complete chip reactor is shown in Fig. 13.

\subsection{Operation of the Chip Reactor}

As the fluid connections are the most critical point when using such a chip reactor, an efficient flange and manifold system is required (Fig. 14). To prevent leakage, the chip reactor is pressed against a PTFE sealing between two PTFE flanges. Reac-
Fig. 13. Photograph of the chip reactor with 19 independent microreaction systems

tant delivery and product withdrawal is realized by capillaries pressed inside holes in the PTFE flange which also acts as a manifold. The Pyrex side of the wafer can be accessed by optical control or analytics. Furthermore, the zones of the microreaction systems allow independent cross-flow of heat exchange fluids on the silicon side of the wafer. The different reaction zones can be efficiently isolated thermally, as shown in Table 2. Thus, it is possible to apply sharp temperature ramps between the reaction zones and to realize very fast quenching of the reaction.

The chip reactor was used for the development of a highly exothermic two-stage process involving hazardous chemicals. In zone 1 , a reaction in aqueous solution took place. In zone 2, an organic reactant was added. Due to the mass transfer enhancement in the microchannels, the multiphase 
Table 2: Results from the independent active temperature control in the different zones of the chip reactor having different parallel microreaction systems (without chemical reaction)

\begin{tabular}{llllllr} 
Experiment & $\begin{array}{l}\text { Zone 1 } \\
\mathrm{T}_{1, \text { in }} \\
{\left[{ }^{\circ} \mathrm{C}\right]}\end{array}$ & $\begin{array}{l}\mathrm{T}_{1 \text {, out }} \\
{\left[{ }^{\circ} \mathrm{C}\right]}\end{array}$ & $\begin{array}{l}\text { Zone 2 } \\
\mathrm{T}_{2, \text { in }} \\
{\left[{ }^{\circ} \mathrm{C}\right]}\end{array}$ & $\begin{array}{l}\mathrm{T}_{2, \text { out }} \\
{\left[{ }^{\circ} \mathrm{C}\right]}\end{array}$ & $\begin{array}{l}\text { Zone 3 } \\
\mathrm{T}_{3, \text { in }}\end{array}$ & $\begin{array}{l}\mathrm{T}_{3, \text { out }} \\
{\left[{ }^{\circ} \mathrm{C}\right]}\end{array}$ \\
\hline 1 & 30.0 & 30.2 & 65.0 & 63.5 & 9.3 & 10.0 \\
2 & 45.0 & 44.4 & 80.0 & 78.3 & 9.5 & 10.3 \\
3 & 60.0 & 58.7 & 90.1 & 89.7 & 9.6 & 11.0 \\
5 & 60.0 & 58.4 & 40.0 & 39.9 & 9.6 & 9.7 \\
6 & 60.0 & 58.5 & 60.1 & 60.1 & 9.6 & 10.2 \\
& 70.0 & 69.8 & 30.0 & 30.2 & 9.6 & 9.6
\end{tabular}

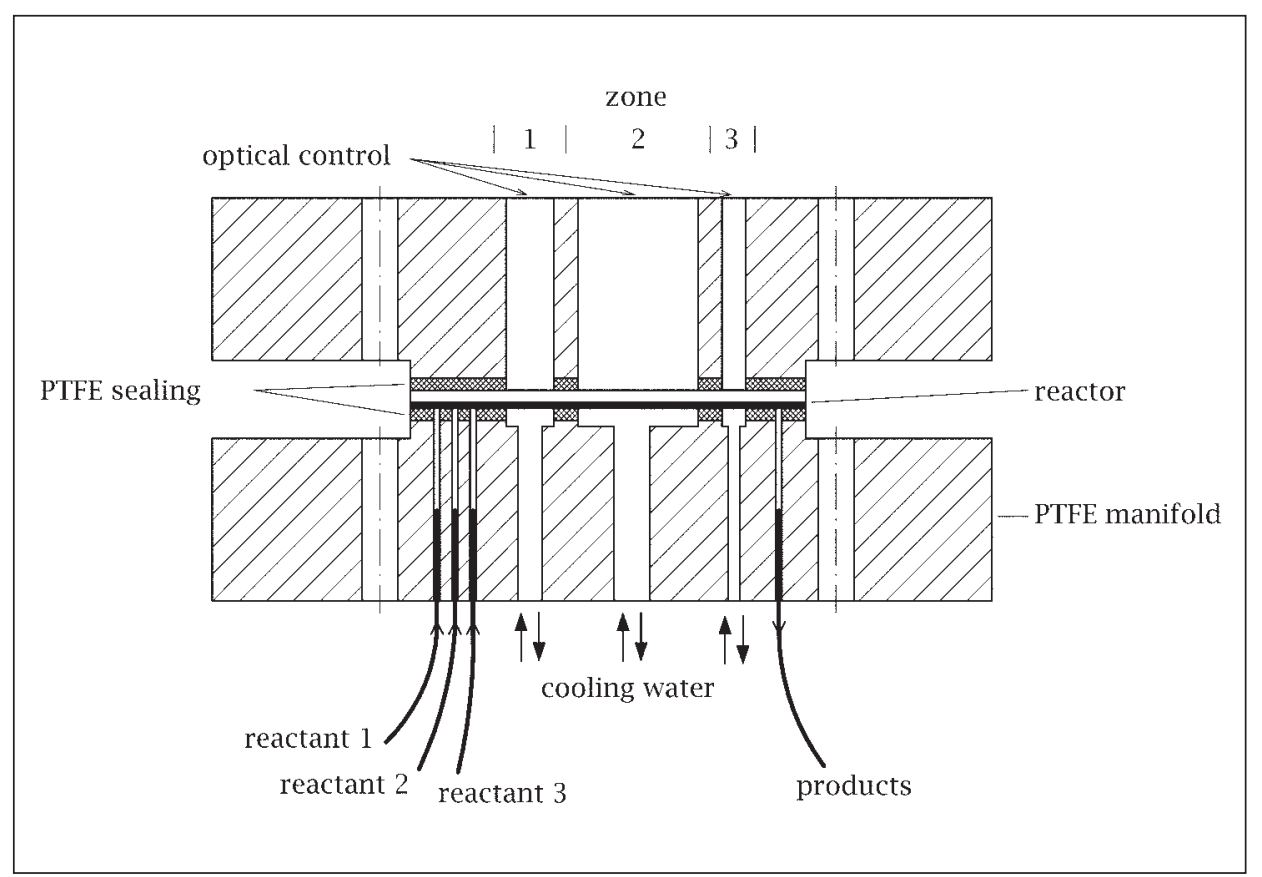

reaction in zone 2 could be carried out efficiently. However, experimental data will be given elsewhere.

\section{Conclusion}

The application of high-throughput methods is fundamentally changing the research paradigms in catalysis and chemical engineering. During the past few years, a number of different concepts have been successfully developed and implemented in academic and industrial research programs. These developments, combined with robotic synthesis and scientific data management strategies, have an enormous impact on the chemical industry and the academic world by dramatically saving the time needed for the discovery and optimization of novel catalytic materials.

The present study showed that miniaturization is one of the key issues in de- signing efficient tools for high-throughput catalyst screening and process development. Different continuously operated miniaturized devices were successfully developed and studied in catalytic reactions, namely microchannel reactors, 'single microchannel' reactors, monolithic reactors, and chip reactors for liquid-phase reactions.

It can be assumed that a number of new developments will be published from academia as well as from industry in the near future. However, there are still many entry barriers to overcome. In many cases, currently available microfabrication technologies limit the development of advanced devices, if not by processing then by the production costs. A better fundamental understanding of microchemical systems is needed to develop new active and passive fluid control elements. CFD simulations have to be routinely applied in the design process. One major point is microscale catalyst preparation, which is still in its infan-
Fig. 14. Sandwich-like flange and manifoldsystem for the parallel microreaction systems from Fig. 12

cy. Furthermore, solutions for high temperature and high pressure operation may prove worthwile in the next years. Of course, high-throughput analytics have to follow the speed and accuracy of the reactor miniaturization. Last but not least, more successful applications need to be published to reduce the sceptical views on microchemical systems still widespread in the scientific community today.

\section{Acknowledgements}

The authors would like to thank the German Federal Ministry of Education and Research (Bundesministerium für Bildung und Forschung) for supporting this project under contract number 03D0068C8 and 03D0068D0, and the Fonds der Chemischen Industrie. T.Z. and D.H. thank the Centre for Microtechnologies, TU Chemnitz, for wafer processing and Cognis Deutschland $\mathrm{GmbH}$ for financial support. P.C. thanks U. Rodemerck, P. Ignaszweski (ACA Berlin) and M. Lucas (TU Darmstadt) for their experimental work in the combicat projects.

Received: August 27, 2002 
[1] M. Lebl, 'Parallel Personal Comments on Classical Papers in Combinatorial Chemistry', J. Comb. Chem. 1999, I (1), 3-24.

[2] X.-D. Xiang, X. Sun, G. Briceño, Y. Lou, K.-A. Wang, H. Chang, W. G. W. Freedman, S.-W. Chen, P. G. Schultz, 'A Combinatorial Approach to Materials Discovery', Science 1995, 268, 1738-1740.

[3] C. Hoffmann, H.-W. Schmidt, F. Schüth, 'A Multipurpose Parallelized 49-Channel Reactor for the Screening of Catalysts: Methane Oxidation as the Example Reaction', J. Catal. 2001, 198, 348-354.

[4] M. Baerns, 'Strategies in the Development of Heterogeneous Catalysts for the Partial Oxidation of Propane by Combinatorial and Evolutionary Methods', Combi2000: Combinatorial Approaches for New Materials Discovery, The Knowledge Foundation, San Diego, California, 2000.

[5] P. Cong, R.D. Doolen, Q. Fan, D.M. Giaquinta, S. Guan, E.W. McFarland, D.M. Poojary, K. Self, H.W. Turner, W.H. Weinberg, 'High-Throughput Synthesis and Screening of Combinatorial Heterogeneous Catalyst Libraries', Angew. Chem. Int. Ed. 1999, 38 (4), 483-488.

[6] B. Jandeleit, D.J. Schaefer, T.S. Powers, H.W. Turner, W.H. Weinberg, 'Combinatorial Materials Science and Catalysis', Angew. Chem. Int. Ed. 1999, 38 (17), 2494-2532.

[7] J.M. Newsam, F. Schüth, 'Combinatorial Approaches As a Component of HighThroughput Experimentation (HTE) in Catalysis Research', Biotechnology and Bioengineering (Combinatorial Chemistry), 1998/1999, 61 (4), 203-216.

[8] A. Holzwarth, H.-W. Schmidt, W.F. Maier, 'Detection of Catalytic Activity in Combinatorial Libraries of Heterogeneous Catalysts by IR Thermography', Angew. Chem. Int. Ed. 1998, 37 (19), 2644-2647.

[9] M. Orschel, J. Klein, H.-W. Schmidt, W.F. Maier, 'Detection of Reaction Selectivity on Catalyst Libraries by Spatially Resolved Mass Spectrometry'. Angew. Chem. Int. Ed. 1999, 38 (18), 2791-2794

[10] S. Senkan, K. Krantz, S. Ozturk, V. Zengin, I. Onal, 'High-Throughput Testing of Heterogeneous Catalyst Libraries Using Array Microreactors and Mass Spectrometry', Angew. Chem. Int. Ed. 1999, 38 (18), 2794-2799.

[11] S.K. Ajmera, C. Delattre, M.A. Schmidt, K.F. Jensen, 'Microfabricated cross-flow chemical reactor for catalyst testing', Sensors and Actuators B 2002, 82, 297-306.

[12] H.S. Bergh, J.R. Engstrom, A. Hagemeyer, C. Lugmair, K. Self, L.V. Erden, P. Cong, S. Guan, Y. Liu, V. Markov, H. Turner, W.H. Weinberg, 'High-Throughput Screening of Combinatorial Heterogeneous Catalyst Libraries', IMRET 4: 4th International Conference on Microreaction Technology, Topical Conference Proceedings, AIChE National Spring Meeting, Atlanta, Georgia, 2000, oral presentation.

[13] S.H. Bergh, S. Guan, 'Chemical processing microsystems, diffusion-mixed microreactors and methods for preparing and using the same', WO 00/51720 to Symyx Technologies, Inc., 1999.

[14] T. Zech, A. Lohf, K. Golbig, T. Richter, D. Hönicke, 'Simultaneous screening of catalysts in microchannels: Methodology and Experimental Setup, Microreaction Technology: Industrial Prospects', IMRET 3: Proceedings of the Third International Conference on Microreaction Technology, Ed. W. Ehrfeld, Springer-Verlag BerlinHeidelberg, 2000, 260-266.

[15] K. Schubert, W. Bier, G. Linder, D. Seidel, 'Herstellung und Test von kompakten Mikrowärmeüberträgern', Chem.-Ing.Tech. 1989, 61 (2), 172-173.

[16] V. Cominos, G. Kolb, K. Drese, R. Zapf, V. Hessel, C. Hoffmann, 'A novel catalyst testing microreactor for heterogeneous gas phase reactions', IMRET 6: 6th International Conference on Microreaction Technology, oral presentation, New Orleans, 2002.

[17] A. Müller, H. Löwe, R. Zapf, V. Hessel, S. Schmitt, 'Fast preparation methods for the coating of microstructures for heterogeneous catalyst screening and experimental proof', IMRET 6: 6th International Conference on Microreaction Technology, poster presentation, New Orleans, 2002

[18] T. Zech, A. Lohf, K. Golbig, T. Richter, D. Hönicke, 'Simultaneous screening of catalysts in microchannels: Methodology and Experimental Setup, Microreaction Technology: Industrial Prospects', IMRET 3: Proceedings of the Third International Conference on Microreaction Technology, Ed. W. Ehrfeld, Springer-Verlag BerlinHeidelberg, April 1999, poster presentation.

[19] T. Zech, 'Miniaturisierte Screening-Systeme für die kombinatorische heterogene Katalyse', Dissertation, Technische Universität Chemnitz, VDI-Verlag Düsseldorf, Reihe 3, Nr. 732, ISBN 3-18373203-3, 2002

[20] B.K. Furlong, J.W. Hightower, T.Y.-L. Chan, A. Sarkany, L. Guczi, '1,3-Butadiene selective hydrogenation over Pd/alumina and $\mathrm{CuPd}$ /alumina catalysts', Appl. Catal. A. General 1994, 117, 41-51.

[21] A. Borgna, B. Moraweck, J. Massardier, A. Renouprez, 'New Supported Palladium-Chromium Catalysts: Characterization and Catalytic Properties', J. Catal. 1991, 128, 99-112.

[22] T. Zech, D. Hönicke, 'Catalyst screening in microchemical systems', First International Symposium on Synthesis, Screening and Sequencing, Frankfurt/Main, 2000, oral presentation.

[23] P. Claus, D. Hönicke, T. Zech, 'Miniaturization of screening devices for the combinatorial development of heterogeneous catalysts', Catalysis Today 2002, 67 (4), 319-339.

[24] T. Zech, D. Hönicke, J. Klein, D. Demuth, S.A. Schunk, 'Vorrichtung zur Analyse und Archivierung von Materialien', WO 02/082094 to hte Aktiengesellschaft, 2001.

[25] T. Zech, D. Hönicke, J. Klein, S.A. Schunk, D. Demuth, 'A novel system architecture for high-throughput primary screening of heterogeneous catalysts', IMRET 5: 5th International Conference on Microreaction Technology, Strasbourg, 2001.

[26] T. Zech, J. Klein, S.A. Schunk, D. Demuth, 'The integrated materials chip for high-throughput experimentation in catalysis research', IMRET 6: 6th International Conference on Microreaction Technology, oral presentation, New Orleans, 2002.

[27] M. Lucas, M. Bonifer, P. Claus, 'Highthroughput screening of catalysts for the total and partial oxidation of hydrocarbons using monolith reactors', Appl. Catal. A, Special Issue 'Combinatorial Catalyst Research', to be published.

[28] T. Zech, D. Hönicke, Chip-Reaktor, EP 1232785 to Cognis Deutschland $\mathrm{GmbH}$, 2001. 